\title{
1 ANALYSIS OF MICROBIAL DIVERSITY IN RAW FISH CEVICHE
}

3 Ramírez-Martínez M. E. ${ }^{1}$; Rodríguez-Castillejos G. C. ${ }^{1}$; Hernández-Jiménez M. C. ${ }^{2}$; Ramírez-

4 Quintanilla L. Y. ${ }^{2}$, Siller-López F. ${ }^{3}$, Acosta-Cruz E. ${ }^{4}$, Martínez-Montoya H. ${ }^{*}$

$5{ }^{1}$ Departamento de Tecnología de Alimentos. U.A.M. Reynosa Aztlán. Universidad

6 Autonoma de Tamaulipas. Reynosa, México.

$7 \quad{ }^{2}$ Departamento de Microbiología. U.A.M. Reynosa Aztlán. Universidad Autónoma de

8 Tamaulipas. Reynosa, México.

$9{ }^{3}$ Programa de Microbiología. Universidad Libre, Seccional Pereira. Pereira, Colombia.

$10{ }^{4}$ Departamento de biotecnología. Facultad de Ciencias Químicas. Universidad Autónoma de

11 Coahuila. Saltillo, México

13 ABSTRACT

14 Ceviche is a traditional dish made from raw fish meat marinated in lime juice without any heat

15 cooking step throughout its preparation process. Although the use of organic acids as

16 antibacterial agents is well known; recent research indicates that lime juice actually can reduce

17 the risk of $V$. parahemolyticus infections but it is ineffective against other potential pathogens.

18 Despite the fact that fresh fish meat is safe; exposed organs including skin, gills and guts

19 represent a potential source of bacterial contamination. In Mexico, diarrheal diseases are caused

20 mainly by contaminated food; it is estimated that almost $67 \%$ of infections are due the presence

21 of bacterial agents mainly in frozen and fresh fish. The main objective of this study was to

22 estimate the taxonomic diversity of microbial species present in ready-to-eat ceviche using a

23 metagenomic approach. Six samples of commercially available ceviche were subjected to DNA 
24 high throughput sequencing and bioinformatics analyses, we identified between 65,000 and

25131,000 reads per sample. The predominant phyla identified through the samples were

26 Proteobacteria, Bacteroidetes and Firmicutes. We discuss the factors involved in the

27 microbiological quality of this kind of raw foods and how they influence the bacterial diversity

28 within the analyzed samples.

30 Keywords: Ceviche; bacteriome; food safety

\section{INTRODUCTION}

33 Ceviche is a traditional dish in many Latin American countries but especially popular in Mexico

34 and Peru. It is made using raw fish meat, marinated few hours in citrus juices in order to

35 eliminate potential pathogens and add the meat a cooked-like flavor and consistency. Lime juice

36 from key limes (Citrus aurantifolia) is one of the most widespread ingredients in its preparation

37 despite the manufacturing region. However, depending of where it is made, other citrus juices

38 such as sour orange and weak organic acids as acetic acid could be used. These products have

39 been reported as effective antibacterial agents due their dissociation inside the bacterial cell into

40 a proton that ultimately will reduce the cytoplasm $\mathrm{pH}$ and an anion that will induce metabolic

41 perturbations in the acidified cytoplasm (Hirshfield, Terzulli et al. 2003). Lime juice has shown

42 antibacterial activity against Vibrio parahemolyticus and V. cholerae when used in raw seafood

43 (Tomotake, Koga et al. 2006) and it exhibited inactivation of common foodborne bacteria such

44 as E. coli O157:H7, S. enteriditis and L. monocytogenes from marinated raw beef (Yang, Lee et

45 al. 2013). 
Bacterial hazards associated to seafood consumption are divided in two groups: the first

47 composed by indigenous bacteria naturally present in the aquatic environment; the second group

48 are introduced bacteria as a result of human, animal or otherwise contamination in the aquatic

49 environment or during the seafood processing and handling. Although ingredients and fish meat

50 used for ceviche may vary by region, in Mexico it is made with either marine fish such as tuna

51 (Thunnus sp.), mullet (Mugil cephalus), sea brass (Dicentrachus labrax) and freshwater species

52 including catfish (Ictalurus punctatus), seabream (Diplodus vulgaris), trout (Oreochromis

53 mykiss) and tilapia (O. niloticus). Fresh fish meat is known to be free of pathogens, however,

54 exposed body structures like skin, gills and guts could increase the risk of foodborne diseases

55 due to parasitic, viral or bacterial contamination. Moreover, poor hygienic handling,

56 transportation and preparation processes could also increase the risk of pathogenic bacteria

57 occurrence and ultimately alter significantly the structure of microbial communities present in

58 seafood. In order to detect such pathogens, traditionally, food microbiology relies on culture-

59 based techniques. However, in addition to their low sensitivity, those techniques need growth

60 conditions that are usually difficult to reproduce in the laboratory, and this could lead to

61 underestimations or bias during the determination of microbial diversity. On the other hand,

62 culture-independent methods based in the sequencing of nucleic acids purified directly from the

63 environmental sample, could improve significantly the limitations exhibited by the classic

64 microbiological methods (De Filippis, Parente et al. 2018). In the present study, we analyzed six

65 ceviche samples through massive sequencing of the 16S V3-V4 rRNA amplicon to estimate the

66 bacterial diversity present in this kind of raw seafood. We obtained between 65,000 and 131,000

67 reads within the sequenced samples, the reads were quality filtered and assembled in contigs.

68 The resulting contigs were compared and identified by homology using the small ribosomal 
69 subunit (SSU) SILVA database v. 132. The predominant phyla found within the samples were

70 Bacteroidetes, Proteobacteria, Firmicutes and Actinobacteria while at genus level,

71 Psychrobacter, Shewanella, Prevotella, Pseudomonas and Pantoea were found the most

72 abundant.

74 MATERIAL AND METHODS

75 Sample collection

76 Ceviche samples, identified as S12, S13, S14 and S15 were obtained from restaurants and street

77 vendors in Reynosa, Mexico. Each sample was transported on ice to the laboratory in sterile

78 plastic bags and stored at $-20 \mathrm{C}$ before DNA purification and further analysis. Additionally,

79 samples S16 and S17 were prepared in the laboratory from $100 \mathrm{~g}$ of chopped mullet and

80 seabream respectively and were marinated with $8 \mathrm{~mL}$ of lime juice two hours before processing.

\section{DNA purification and sequencing}

82 Ceviche samples were homogenized in $0.9 \%$ sterile saline solution before DNA purification.

83 Total genomic DNA was recovered from 0.25 grams of sample using a modified chloroform-

84 ethanol procedure using $1 \mathrm{~mL}$ of lysis buffer (0.5M EDTA, 10\% SDS) and precipitated in two

85 steps with isopropanol and ethanol. Finally, it was diluted in $50 \mu \mathrm{L}$ of AE buffer (Qiagen,

86 Valencia, CA). Prior the library preparation, DNA quality control was performed with

87 Bioanalyzer (Agilent, Santa Clara, CA) to determine the size of DNA fragments and its

88 abundance throughout the samples. DNA pair-ended library was performed according to

89 Illumina's Multiplexing Sample Preparation Guide (Illumina, San Diego, CA), the DNA was

90 fragmented, end repaired, A' tagged, ligated to 357-F and CD-R adaptors, size-selected, and

91 enriched with 25 cycles of PCR during which an index was incorporated to the samples (Table 
92 S1). The resulting libraries were subjected to Illumina MiSeq (2x300) pair-ended sequencing at

93 the Laboratory of Genomic Services of the National Laboratory of Genomics for Biodiversity

94 (LANGEBIO) (Irapuato, Mexico).

\section{Sequencing data analyses}

96 Taxonomic labels were assigned to the raw reads in each sample by homology with a local $16 \mathrm{~S}$

97 rRNA database using Kraken 2 (Wood and Salzberg 2014) in the Illumina BaseSpace Sequence

98 Hub (https://basespace.illumina.com). Additionally, sequencing reads were assembled in contigs

99 and quality filtered following the Mothur pipeline (Schloss, Westcott et al. 2009) to assess

100 species richness and alpha diversity.

101 Contigs were compared and identified by homology with the ribosomal small subunit (SSU)

102 SILVA Database v. 132 (Quast, Pruesse et al. 2013). Aligned sequences were screened and to 103 non-bacterial and chimeric sequences were removed. The alpha diversity for each sample was

104 reported as observed and calculated using the Chao1, ACE and Simpson's diversity metrics and 105 visualized throughout the Microbiome Analyst Portal (https://www.microbiomeanalyst.ca)

106 (Dhariwal, Chong et al. 2017). Finally, metagenomeSeq (Paulson, Stine et al. 2013) was used to

107 find differential abundance of microorganisms at different taxonomic levels among commercial 108 and control samples.

\section{RESULTS}

111 Sequenced reads were deposited and are available in the NCBI sequence depository under the

112 Raw Fish Ceviche Bacterial Diversity BioProject (ID: PRJNA512206). We identified between

11365,000 and 131,000 reads among the sequenced samples. According to the taxonomic labels

114 given by both pipelines, Kraken and Mothur, the predominant phyla across the ceviche samples 
were Proteobacteria, Actinobacteria, Firmicutes and Bacteroidetes. Additionally, we found

116 several genera with low count of reads, Chloroflexi, Cyanobacteria, Fusobacteria and

117 Gemmatimonadetes. At genus level, Kraken analysis showed high predominance of

118 Bacteroidetes followed by Firmicutes in S12 but S13 had high presence of Firmicutes, whereas

$119 \mathrm{~S} 14, \mathrm{~S} 15, \mathrm{~S} 16$ and S17 showed predominance of bacteria belonging to the phylum Proteobacteria

120 (Table 1).

121 At genus level, in S12 we have found a high prevalence of Bacteroidetes, mainly represented by

122 Prevotella species (58\%) such as $P$. oris, $P$. dentalis, $P$. fusca, $P$. denticola; the second largest

123 group of bacteria in S12 belongs to the phylum Firmicutes and had homologous sequences to

124 species such as Faecalibacterium prausnitzii, Eubacterium rectale, Ruminococcus bicirculans,

125 Selenomonas sputigena and S. ruminantium, Megaspharea elsdenii and in less frequency, we

126 found S. aureus and Streptococcus sp. In S13 we observed a high relative abundance (RA) of not

127 assigned bacterial genus (72.2\%) but also we found Psychrobacter (9.34\%), Rothia (5.08\%) and

128 Paracoccus (3.7\%) additionally represented in less than 2\% of RA, Enterobacteria, Pantoea,

129 Propionibacterium and Shewanella. Sample S15 had 0.95\% of RA of Empedobacter and

130 Rheinheimera. S14 had a high presence of Pantoea (50\%) and genera such as Rosenbergiella

131 (16.95\%), unclassified Enterobacteriaceae (11.96\%) and Weisella (5.46\%). In less percentage,

132 we found Myroides, Pseudomonas, Chryseobacterium, Rhizobium, Buttiauxella,

133 Sphingobacterium, Soonwooa, Rothia and Pseudochrobacterium. Non-commercial samples, S16

134 and S17 were only fish meat marinated with lime juice. Whereas S16 exhibited Psychrobacter

135 (16.45\%), Pseudomonas (3.5\%), Propionibacterium (1.9\%), Shewanella, Roseiflexus,

136 Flavobacterium, Acinetobacter, Rothia, Bradirhizobium and Aeromonas on the other hand, S17

137 showed Aeromonas (19.13\%), Shewanella (8.77\%), Propionibacterium (8.09\%), Psychrobacter 
$138(3.8 \%)$ and less represented, Corynebacterium, Acinetobacter, Roseiflexus, Pseudomonas,

139 Flavobacterium and Buttiauxella.

140 Accordingly, to the Kraken analysis, Mothur analyses revealed a high abundance of

141 Bacteroidetes in the sample S12 but we found a relatively low abundance of Firmicutes, and the

142 most abundant phyla in S13, S14, S15, S16 and S17 were Proteobacteria, Actinobacteria,

143 Firmicutes, Chloroflexi and Armatimonadetes (Figure 1 and Table S2).

144 At genus level, we found mostly Prevotella in S12 (0.995) while in S13-S15 a higher diversity

145 was observed (Figure 2). The rarefaction richness plot observed in samples was not saturated in

146 the ceviche samples but it clearly indicated that sample S12 had the lowest richness while S17

147 showed the highest number of OTU's. (Figure 3 and Table S3).

148 Alpha diversity was assessed through observed and estimated analyses using the Mothur data.

149 We did not found significant differences in observed diversity among samples $(P=0.29724)$.

150 however, according to the rarefaction analysis, S12 and S17 had the lowest and highest diversity

151 with 60 and 197 OTU's respectively (Table 2). In addition to the observed count of species we

152 calculated the alpha diversity trough $\operatorname{ACE}(P=0.71295)$, Chaol $(P=0.98386)$ and Simpson's

153 diversity indexes $(P=0.2646)$ (Table 2).

154 Correlation and clustering visualization analysis showed that based on the microbial profiles,

155 laboratory-made samples S16 and S17 were clustered together whereas commercial samples

156 formed the other cluster. It is observed that S16 and S17 samples were strongly positively

157 correlated to the presence of bacteria belonging to the Psychrobacter, Pseudomonas,

158 Bradyrhizobium, Shewanella, Propionibacterium, Corynebacterium, Aeromonas,

159 Flavobacterium, Acinetobacter and Vagococcus genera which do not suggest evidence of human

160 contamination (Figure 4). On the other hand, S12, S13 and S14 had a strong correlation with 
161 bacterial classes typically associated to human contamination such as Enterobacteriales

162 Lactobacillales, Bacteroidales and Rhodobacterales. We found that Enterobacteriaceae $(P=$

163 2.63E-4), Prevotellaceae $(P=0.005)$ and Leuconostocaceae $(P=0.005)$ family members had a

164 significant higher abundance in ceviche samples from restaurants than in the laboratory made

165 samples whereas Moraxellaceae was more slightly abundant in the control samples $(P=0.02)$

166 (Table S4).

167

\section{DISCUSSION}

169 Bacterial agents found in the tested ceviche samples showed high diversity of taxa across the

170 samples, as stated previously. In general, bacterial in seafood belong to two different groups,

171 native marine or freshwater bacteria present in naturally in the aquatic environment and those

172 bacteria present due exogenous contamination of human or animal sources.

173 Among the native bacteria identified in this study we found Soonwooa, a member of the

174 Flavobacteriaceae family is a previously isolated microorganism from the yellow sea waters

175 from Korea (Joung, Song et al. 2010), it was found mainly in S14 and S15. Nowadays, two

176 species of Soonwooa, $S$. sp. and $S$. buanensis have been described in tissues from tilapia $(O$.

177 niloticus) reared in dams in Mexico (Soto-Rodriguez, Cabanillas-Ramos et al. 2013) and in duck

178 meat products from Korea (Kim, H.I. et al. 2016) but were not identified as pathogenic agents.

179 Within the Flavobacteriaceae family we also identified reads hitting to the gram-negative

180 Chryseobacterium in S13 and S15, although we were not able to identify the bacteria at species

181 level, its close relatives, previous reports mention that $C$. meningosepticum has been associated

182 to foodborne septicemia due consumption of raw seafood (Kim, H.I. et al. 2016) and $C$.

183 indologenes which is widely spread in soil and plants rarely was associated to human disease 
184 (Imataki and Uemura 2017). This last example could be present in ceviche since the samples

185 involved raw vegetables in its preparation, however, further analyses are required to elucidate the

186 origin of Chryseobacterium species present in raw seafood.

187 The genus Rheinheimera, found in S14 is widely distributed in freshwater, saltwater and soil

188 environments. Is a Gram-negative bacterium that presents antimicrobial activity in some strains

189 (Chen, Lin et al. 2010). Additionally, other bacteria naturally associated to aquatic environments

190 such as Shewanella, Psychrobacter, Vagococcus, Buttiauxella and Novosphingobium were

191 found, although they do not represent a potential source of foodborne illness

192 Vegetables in ceviche, including cilantro, onion, tomato, pepper and avocado are usually

193 consumed raw; in ceviche, they represent a major source of pathogenic bacteria that is associated

194 to foodborne illness. In this study the ceviche samples tested had a variety of produce ingredients

195 that constituted a factor of variability in the results. Among exogenous bacteria, we identified

196 several members of the Enterobacteriaceae genus such as Pantoea, Rosenbergiella and

197 Morganella. Pantoea, primarly found in S15 but also in S13, these genera are known to be

198 associated as a pathogen or epiphytic bacteria in edible vegetables, e.g P. agglomerans in tomato

199 and onion (Oie, Kiyonaga et al. 2008, Nadarasah and Stavrinides 2014) which are main

200 ingredients of Mexican made ceviche. P. agglomerans, has also been associated to soft bone

201 infections [41], although this opportunistic bacterium is not known to be a foodborne pathogen it

202 has been associated as a contaminant in powered infant formula milk (Mardaneh and Dallal

203 2013). We hypothesized that Pantoea sp. found in ceviche have its origin in the vegetables used

204 in the dish preparation since it was almost undetectable in the laboratory made samples S16 and

205 S17 respectively. 
Rothia sp., found in a highest frequency in S13 may cause a significant number of infections to

207 immunocompromised hosts despite they are part of the normal microbiome of human

208 oropharynx and respiratory tract (Ramanan, Barreto et al. 2014), it is possible that Rothia in

209 ceviche have its origin from anthropogenic contamination during its preparation.

210 The genus Weissella, found primarily in S15 but also in S13 is a gram-positive cocco-bacilli,

211 often misidentified by traditional microbiological methods but commonly found in fermented

212 foods, including vegetables, meat, fish and raw milk. Although, this genus is rarely a known

213 cause of human infections. Conversely, few Weissella strains may act as probiotics due its

214 antimicrobial activity e.g. W. hellenica isolated from flounder intestine act as a defensive agent

215 against host pathogens such as Edwardsiella, Pasteurella, Aeromonas and Vibrio (Cai, Benno et

216 al. 1998, Abriouel, Lerma et al. 2015).

217 Aeromonas, a genus found in high presence in S17, but present in all the tested samples is one of

218 the etiological agents associated to disease-causing pathogen of fish and responsible of infectious

219 complications in humans. This genus can be isolated from almost every ecological niche in earth,

220 including aquatic habitats, fish, food and soils and it can be present in ceviche due multiple

221 factors and represented by one or more species. In food, Aeromonas strains have been widely

222 reported in dairy products, specifically unpasteurized milk, yogurt and cheese from diverse

223 geographical origins.

224 Prevotella, was detected in all samples except S15 and S17, this agent is known to be a part of

225 human gut, oral and vaginal microbiota, this bacterium is often an indicator of fecal

226 contamination of water (Fogarty and Voytek 2005, Koskey, Fisher et al. 2014). However, in

227 ceviche the high presence of this agent could be associated to low hygienic conditions in produce 228 handling. 
Pseudomonas, a naturally widespread genus is a potential human pathogen previously reported

230 in spinach, lettuce and red cabbage, it was found in every sample and probably its presence is

231 due either contaminated vegetables and contamination of fish meat. In the present study, we did

232 not found etiological agents associated to freshwater fish species outbreaks of foodborne illness

233 such as Salmonella, Escherichia coli, Clostridium, Staphylococcus or Bacillus, this study

234 presents the first description of the bacterial diversity in raw fish food using a metagenomic

235 approach. However, as observed, microbiological quality of raw foods, depends on many factors

236 bacterial diversity in raw fish ceviche increases as raw vegetables are added. Further analyses are

237 needed in order to examine the dynamics of bacterial communities in ready-to-eat raw seafood.

239 Declaration of competing interest

240 The authors declare no competing interests

242 Acknowledgments

243 Authors thank to the Secretaría de Educación Pública of Mexico for the funding given to this

244 study through the PRODEP-NPTC fellowship granted to Humberto Martinez Montoya and

245 Myriam Elizabeth Ramirez Martinez (UAT-PTC-211)

247 References

248 Abriouel, H., L. L. Lerma, C. Casado Munoz Mdel, B. P. Montoro, J. Kabisch, R. Pichner, G. S.

249 Cho, H. Neve, V. Fusco, C. M. Franz, A. Galvez and N. Benomar (2015). "The controversial

250 nature of the Weissella genus: technological and functional aspects versus whole genome 
251 analysis-based pathogenic potential for their application in food and health." Front Microbiol 6:

2521197.

253 Cai, Y., Y. Benno, T. Nakase and T. K. Oh (1998). "Specific probiotic characterization of

254 Weissella hellenica DS-12 isolated from flounder intestine." J Gen Appl Microbiol 44(5): 311-

255316.

256 Chen, W. M., C. Y. Lin, C. C. Young and S. Y. Sheu (2010). "Rheinheimera aquatica sp. nov.,

257 an antimicrobial activity producing bacterium isolated from freshwater culture pond." $\underline{\mathrm{J}}$

258 Microbiol Biotechnol 20(10): 1386-1392.

259 De Filippis, F., E. Parente and D. Ercolini (2018). "Recent Past, Present, and Future of the Food

260 Microbiome." Annu Rev Food Sci Technol 9: 589-608.

261 Dhariwal, A., J. Chong, S. Habib, I. L. King, L. B. Agellon and J. Xia (2017).

262 "MicrobiomeAnalyst: a web-based tool for comprehensive statistical, visual and meta-analysis of

263 microbiome data." Nucleic Acids Res 45(W1): W180-W188.

264 Fogarty, L. R. and M. A. Voytek (2005). "Comparison of bacteroides-prevotella 16S rRNA

265 genetic markers for fecal samples from different animal species." Appl Environ Microbiol

266 71(10): 5999-6007.

267 Hirshfield, I. N., S. Terzulli and C. O'Byrne (2003). "Weak organic acids: a panoply of effects on

268 bacteria." Sci Prog 86(Pt 4): 245-269.

269 Imataki, O. and M. Uemura (2017). "Chryseobacterium indologenes, a possible emergent

270 organism resistant to carbapenem antimicrobials after stem cell transplantation." Clin Case Rep

$271 \quad 5(1): 22-25$. 
272 Joung, Y., J. Song, K. Lee, H. M. Oh, K. Joh and J. C. Cho (2010). "Soonwooa buanensis gen.

273 nov., sp. nov., a member of the family Flavobacteriaceae isolated from seawater." Int J Syst Evol

274 Microbiol 60(Pt 9): 2061-2065.

275 Kim, H. J., Y. H.I., H. J. Lee, S. Jung, J. H. Kwon, K. N. Heo and C. Jo (2016). "Identification of

276 microorganisms in duck meat products available in Korea and the effect of high hydrostatic

277 pressure." Korean Journal for Food Science of animal Resources 34(2): 283-288.

278 Koskey, A. M., J. C. Fisher, A. M. Eren, R. Ponce-Terashima, M. G. Reis, R. E. Blanton and S.

279 L. McLellan (2014). "Blautia and Prevotella sequences distinguish human and animal fecal

280 pollution in Brazil surface waters." Environ Microbiol Rep 6(6): 696-704.

281 Mardaneh, J. and M. M. Dallal (2013). "Isolation, identification and antimicrobial susceptibility

282 of Pantoea (Enterobacter) agglomerans isolated from consumed powdered infant formula milk

283 (PIF) in NICU ward: First report from Iran." Iran J Microbiol 5(3): 263-267.

284 Nadarasah, G. and J. Stavrinides (2014). "Quantitative evaluation of the host-colonizing

285 capabilities of the enteric bacterium Pantoea using plant and insect hosts." Microbiology $160(\mathrm{Pt}$

286 3): 602-615.

287 Oie, S., H. Kiyonaga, Y. Matsuzaka, K. Maeda, Y. Masuda, K. Tasaka, S. Aritomi, A.

288 Yamashita and A. Kamiya (2008). "Microbial contamination of fruit and vegetables and their

289 disinfection." Biol Pharm Bull 31(10): 1902-1905.

290 Paulson, J. N., O. C. Stine, H. C. Bravo and M. Pop (2013). "Differential abundance analysis for

291 microbial marker-gene surveys." Nat Methods 10(12): 1200-1202.

292 Quast, C., E. Pruesse, P. Yilmaz, J. Gerken, T. Schweer, P. Yarza, J. Peplies and F. O. Glockner

293 (2013). "The SILVA ribosomal RNA gene database project: improved data processing and web-

294 based tools." Nucleic Acids Res 41(Database issue): D590-596. 
Ramanan, P., J. N. Barreto, D. R. Osmon and P. K. Tosh (2014). "Rothia bacteremia: a 10-year experience at Mayo Clinic, Rochester, Minnesota." J Clin Microbiol 52(9): 3184-3189.

297 Schloss, P. D., S. L. Westcott, T. Ryabin, J. R. Hall, M. Hartmann, E. B. Hollister, R. A.

298 Lesniewski, B. B. Oakley, D. H. Parks, C. J. Robinson, J. W. Sahl, B. Stres, G. G. Thallinger, D.

299 J. Van Horn and C. F. Weber (2009). "Introducing mothur: open-source, platform-independent,

300 community-supported software for describing and comparing microbial communities." Appl

301 Environ Microbiol 75(23): 7537-7541.

302 Soto-Rodriguez, S. A., J. Cabanillas-Ramos, U. Alcaraz, B. Gomez-Gil and J. L. Romalde

303 (2013). "Identification and virulence of Aeromonas dhakensis, Pseudomonas mosselii and

304 Microbacterium paraoxydans isolated from Nile tilapia, Oreochromis niloticus, cultivated in

305 Mexico." J Appl Microbiol 115(3): 654-662.

306 Tomotake, H., T. Koga, M. Yamato, A. Kassu and F. Ota (2006). "Antibacterial activity of citrus 307 fruit juices against Vibrio species." J Nutr Sci Vitaminol (Tokyo) 52(2): 157-160.

308 Wood, D. E. and S. L. Salzberg (2014). "Kraken: ultrafast metagenomic sequence classification 309 using exact alignments." Genome Biol 15(3): R46.

310 Yang, J., D. Lee, S. Afaisen and R. Gadi (2013). "Inactivation by lemon juice of Escherichia coli

311 O157:H7, Salmonella Enteritidis, and Listeria monocytogenes in beef marinating for the ethnic

312 food kelaguen." Int J Food Microbiol 160(3): 353-359. 
bioRxiv preprint doi: https://doi.org/10.1101/2020.06.24.170217; this version posted June $25,2020$. The copyright holder for this preprint (which was not certified by peer review) is the author/funder. All rights reserved. No reuse allowed without permission.

\begin{tabular}{|c|c|c|c|c|c|c|c|c|c|c|c|c|}
\hline & \multicolumn{2}{|c|}{ S12 } & \multicolumn{2}{|c|}{ S13 } & \multicolumn{2}{|c|}{ S14 } & \multicolumn{2}{|c|}{ S15 } & \multicolumn{2}{|l|}{ S16 } & \multicolumn{2}{|c|}{ S17 } \\
\hline Total Reads & & 8922 & & 0284 & 353 & & & 3376 & 9450 & & & 6900 \\
\hline Phylum & $\%$ & Reads & $\%$ & Reads & $\%$ & Reads & $\%$ & Reads & $\%$ & Reads & $\%$ & Reads \\
\hline Actinobacteria & 1.1 & 702 & 0.9 & 194 & 2.5 & 90 & 0.2 & 119 & 3.0 & 286 & 3.3 & 888 \\
\hline Bacteroidetes & 57.2 & 33725 & 2.4 & 489 & 12.4 & 440 & 2.7 & 1184 & 5.1 & 489 & 1.0 & 274 \\
\hline Chloroflexi & 0.02 & 12 & 0.1 & 27 & 0.8 & 31 & 0 & 4 & 1.0 & 103 & 0.4 & 121 \\
\hline Cyanobacteria & 0.02 & 14 & 0.1 & 33 & 1.3 & 49 & 0.0 & 13 & 0.9 & 94 & 0.2 & 74 \\
\hline Firmicutes & 36.8 & 21718 & 86.7 & 17604 & 32.5 & 1151 & 32.7 & 14201 & 13.2 & 1250 & 4 & 1076 \\
\hline Fusobacteria & 0 & 1 & 0.02 & 6 & 0.3 & 12 & 0 & 6 & 0.1 & 12 & 0.1 & 30 \\
\hline Gemmatimonadetes & 0 & 1 & 0.02 & 5 & 0.0 & 1 & 0 & 3 & 0.0 & 4 & 0.0 & 13 \\
\hline Proteobacteria & 4.2 & 2510 & 8.5 & 1738 & 46.5 & 1646 & 63.9 & 27725 & 74.4 & 7038 & 89.9 & 24198 \\
\hline
\end{tabular}

Table 2. Alpha diversity observed and calculated for analyzed samples.

321

\begin{tabular}{|l|l|l|l|l|l|l|l|l|l|}
\cline { 2 - 10 } \multicolumn{2}{c|}{} & \multicolumn{2}{c|}{ Chao1 } & \multicolumn{2}{c|}{ ACE } & \multicolumn{2}{c|}{ Simpson } & \multicolumn{2}{l|}{ Observed } \\
\hline Sample type & Sample ID & Value & SE & Value & SE & Value & SE & Value & SE \\
\hline Commercial & S12 & 8.33 & 0.91 & 11.27 & 1.79 & 0.009 & NA & 60 & NA \\
\hline Commercial & S13 & 24.75 & 4.19 & 27.82 & 2.71 & 0.45 & NA & 132 & NA \\
\hline Commercial & S14 & 17.2 & 0.61 & 17.94 & 1.85 & 0.04 & NA & 177 & NA \\
\hline Commercial & S15 & 18.5 & 2.57 & 19.6 & 1.9 & 0.67 & NA & 146 & NA \\
\hline Control & S16 & 23 & 0.48 & 23.26 & 2.31 & 0.51 & NA & 148 & NA \\
\hline Control & S17 & 22 & 0.48 & 22.38 & 1.92 & 0.71 & NA & 197 & NA \\
\hline
\end{tabular}

\section{Figures}


bioRxiv preprint doi: https://doi.org/10.1101/2020.06.24.170217; this version posted June 25, 2020. The copyright holder for this preprint (which was not certified by peer review) is the author/funder. All rights reserved. No reuse allowed without permission.

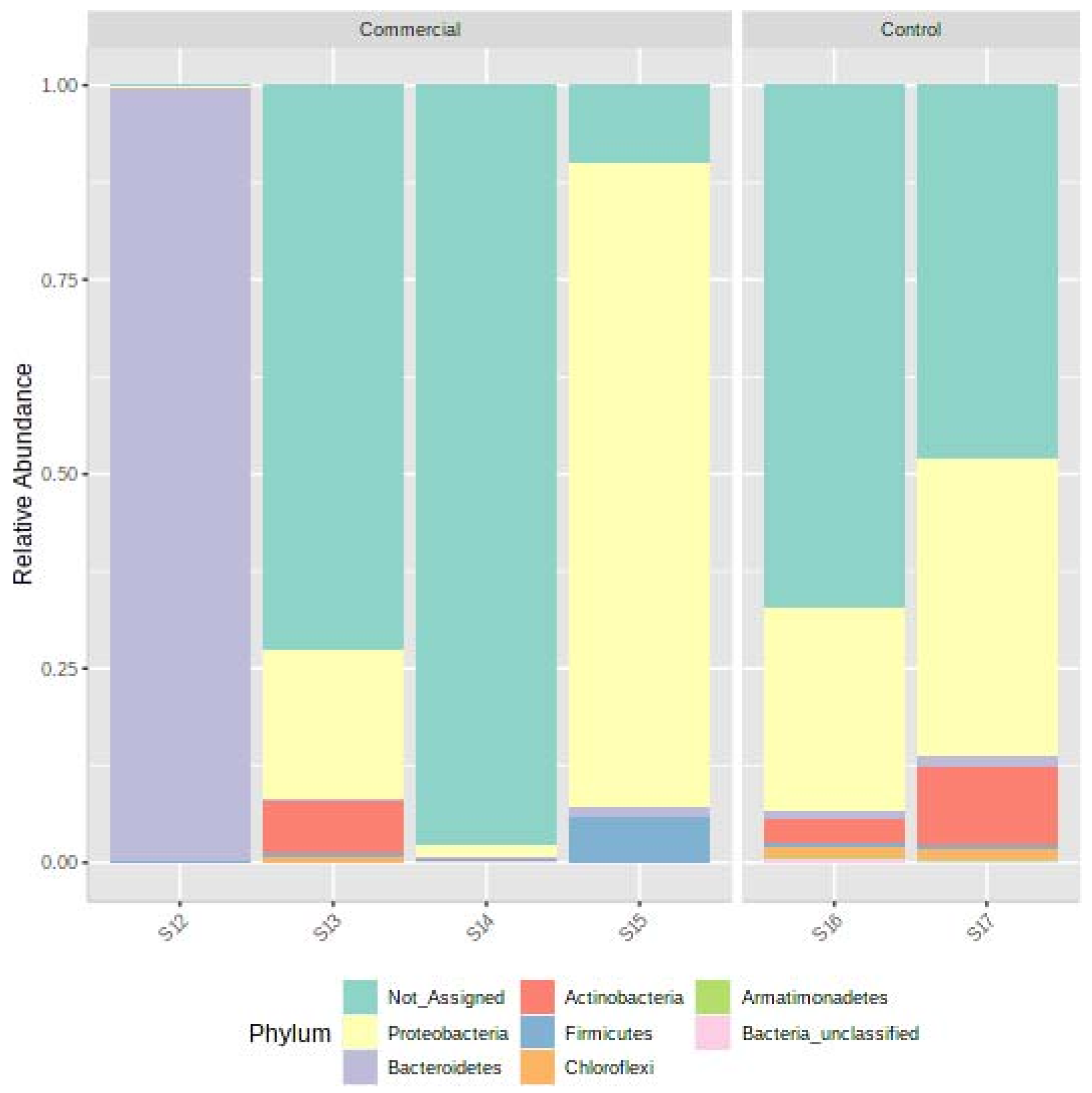

Fig 1. Dominant phyla across analyzed ceviche samples. 
bioRxiv preprint doi: https://doi.org/10.1101/2020.06.24.170217; this version posted June 25, 2020. The copyright holder for this preprint (which was not certified by peer review) is the author/funder. All rights reserved. No reuse allowed without permission.

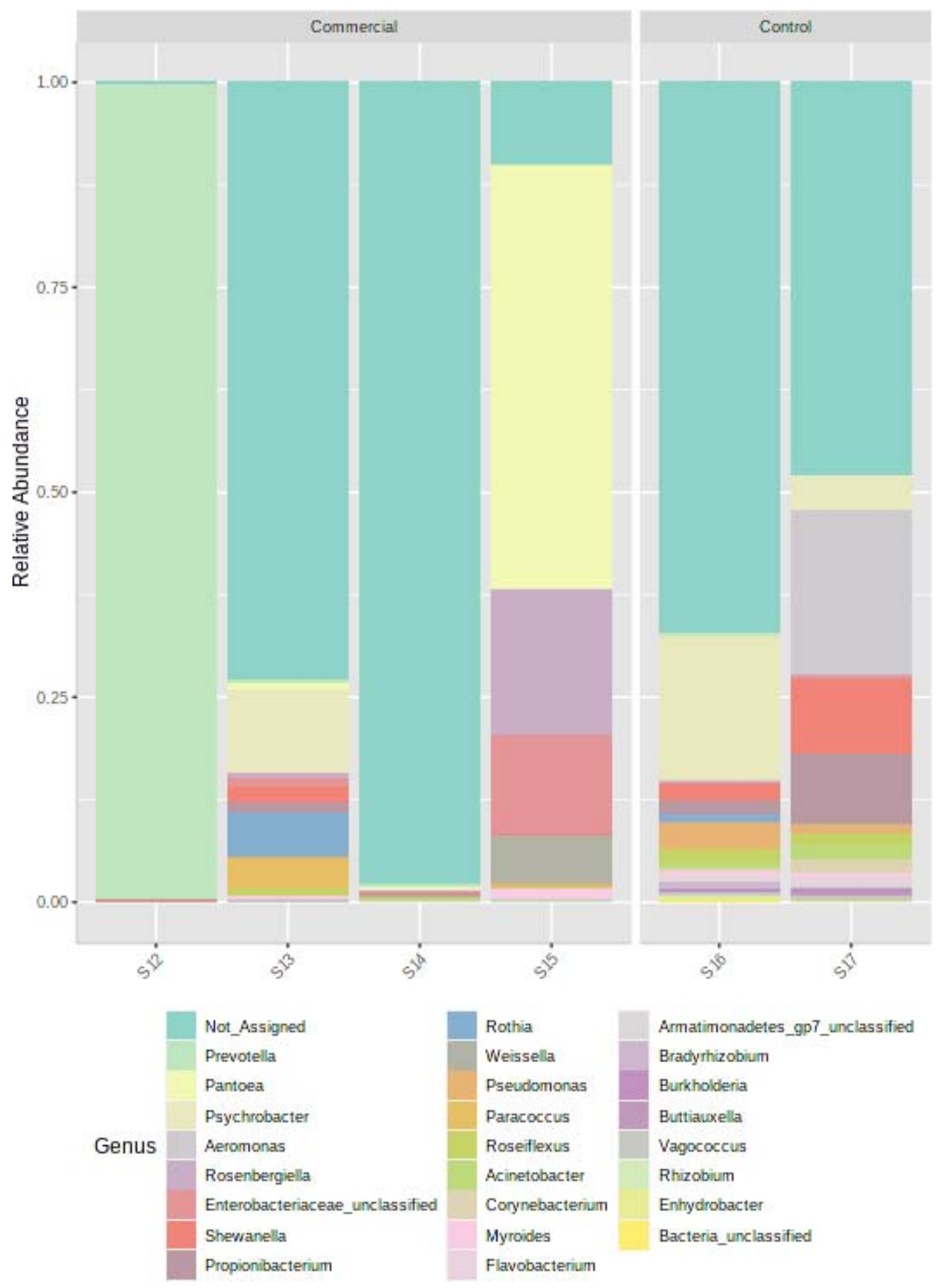

Figure 2. Relative abundance of bacterial genera within the Ceviche samples. 
bioRxiv preprint doi: https://doi.org/10.1101/2020.06.24.170217; this version posted June 25, 2020. The copyright holder for this preprint (which was not certified by peer review) is the author/funder. All rights reserved. No reuse allowed without permission.

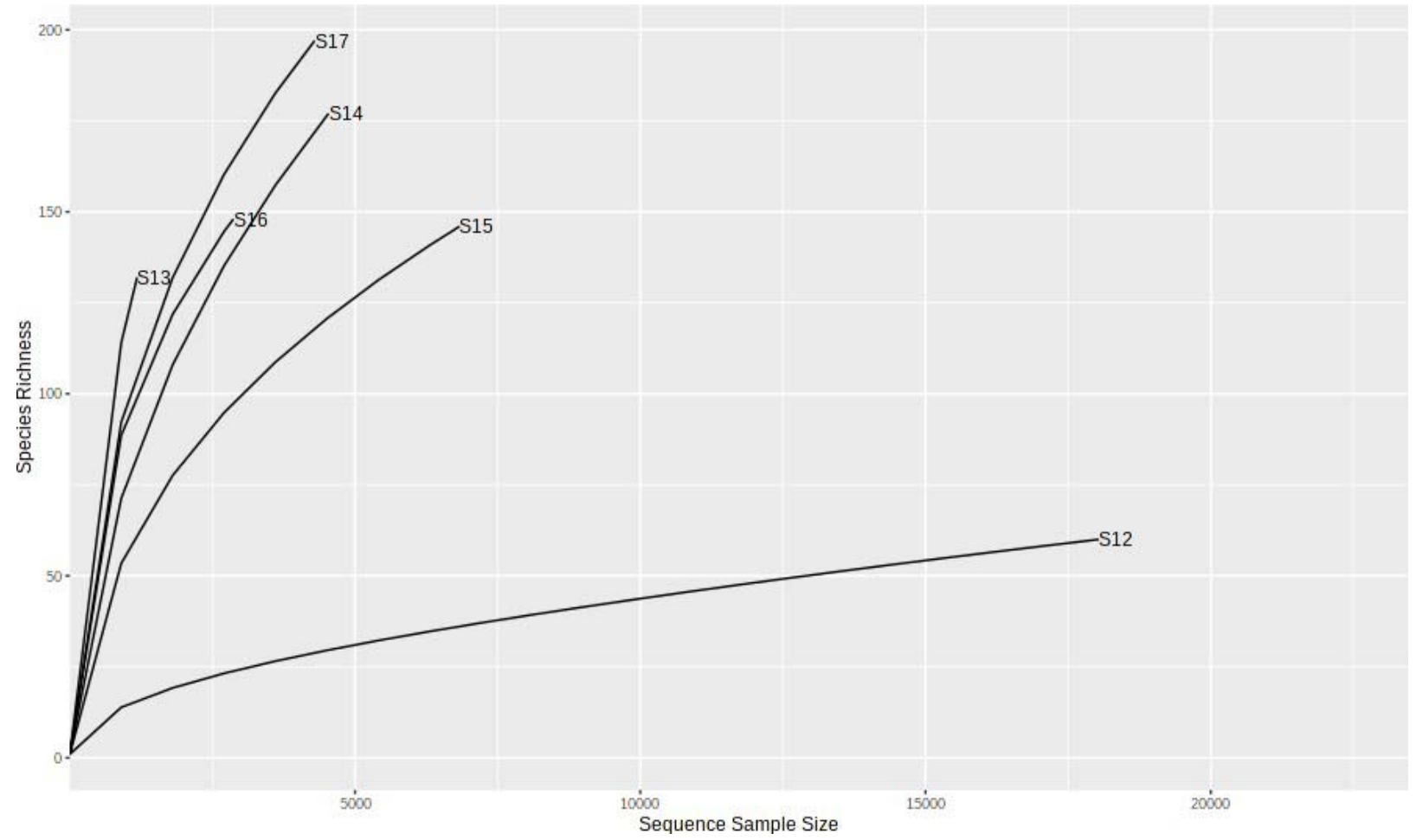

Figure 3. Richness rarefaction plot of analyzed ceviche samples. 
bioRxiv preprint doi: https://doi.org/10.1101/2020.06.24.170217; this version posted June 25, 2020. The copyright holder for this preprint (which was not certified by peer review) is the author/funder. All rights reserved. No reuse allowed without permission.

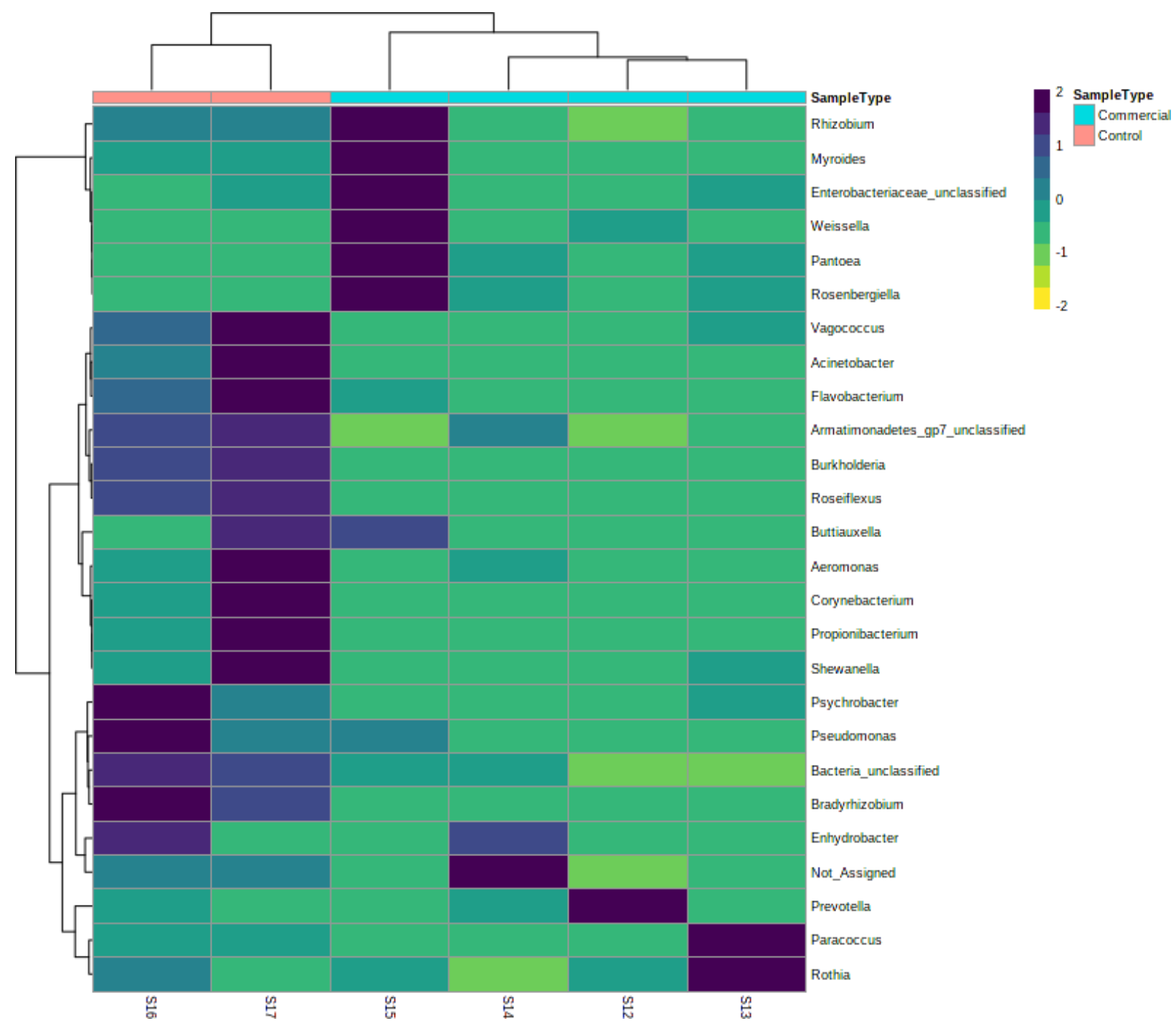

334 Figure 4. Hierarchical clustering and heatmap visualization of commercial and laboratory made samples at genus level. 